\title{
Smac-mimetic enhances antitumor effect of standard chemotherapy in ovarian cancer models via Caspase 8-independent mechanism
}

\author{
Lidia F. Hernandez ${ }^{1}$, Angie B. Dull², Soumya Korrapati ${ }^{1}$ and Christina M. Annunziata ${ }^{1}$
}

\begin{abstract}
Ovarian cancer is the most lethal gynecological cancer in the US. Standard treatment consists of surgery followed by chemotherapies relying on apoptotic tumor cell death. Most women with advanced stage disease will relapse, suggesting that this disease is characterized by primary and acquired resistance to chemotherapy, and novel approaches to treatment are greatly needed. Low Caspase 8 expression levels in ovarian cancers correlate with resistance to apoptotic chemotherapy, and a subpopulation of patients with low Caspase 8 levels exhibit poorer overall survival after standard-of-care treatment. We hypothesized that low Caspase 8 function reduces the ability of cancer cells to undergo apoptosis when exposed to standard chemotherapy and that second mitochondria-derived activator of caspases (Smac)-mimetics could increase cell death in combination with chemotherapy. Here we show that combination treatment with a Smac-mimetic can target tumor cells with low Caspase 8 and induce necroptotic cell death. We investigated the in vitro effect of Smac-mimetic added to carboplatin and paclitaxel treatment of ovarian cancer cells expressing wild type and low Caspase 8 levels, which resulted in a 2-4-fold enhancement of cell death. Mice bearing subcutaneous or intraperitoneal ovarian xenografts showed greater aggressiveness of Caspase 8deficient versus wild-type tumors; combined in vivo treatment with chemotherapy and Smac-mimetic resulted in $>50 \%$ decrease in low Caspase 8 xenograft growth, as well as significantly enhanced overall survival, especially when given simultaneously with paclitaxel. Surprisingly, Smac-mimetic on the same day as carboplatin decreased mouse survival compared to when it was given on a sequential day of treatment. The antagonism was associated with a decrease in DNA damage markers, emphasizing the importance of optimizing timing of drug administration. Clinical validation of such approaches is needed to increase the effectiveness of current standard ovarian cancer treatment.
\end{abstract}

\section{Introduction}

Ovarian cancer is expected to produce 21,750 new cases and 13,940 deaths in the US in $2020^{1}$. Since the introduction of platinum/taxane therapies in the 1990s, there have been few improvements in standard chemotherapy regimens especially for women with homologous recombination-proficient cancers, and 5-year survival for

Correspondence: Christina M. Annunziata (annunzic@mail.nih.gov)

'Women's Malignancies Branch, Center for Cancer Research, National Cancer Institute, National Institutes of Health, Bethesda, MD, USA

${ }^{2}$ Clinical Pharmacodynamics Program, Frederick National Laboratory/Leidos, Frederick, MD, USA

Edited by A. Rufini advanced disease remains at $47 \%$ (http://seer.cancer.gov/ statfacts/html/ovary.html). Standard treatments typically rely on apoptotic cell death for targeting tumor cells, an effective primary treatment, but most women relapse and exhibit acquired resistance to chemotherapy, highlighting the need for secondary lines of treatment in order to improve outcome.

Recent molecular characterization of ovarian cancers has identified subgroups, adding prognostic value as well as informing new therapeutic approaches ${ }^{2,3}$. We recently established the role of nuclear factor (NF)-кB on inflammation and tumor cell survival in ovarian cancer $^{4}$ and demonstrated the dual role of the pro-

\section{(c) The Author(s) 2021}

(c) (i) Open Access This article is licensed under a Creative Commons Attribution 4.0 International License, which permits use, sharing, adaptation, distribution and reproduction cc) in any medium or format, as long as you give appropriate credit to the original author(s) and the source, provide a link to the Creative Commons license, and indicate if changes were made. The images or other third party material in this article are included in the article's Creative Commons license, unless indicated otherwise in a credit line to the material. If material is not included in the article's Creative Commons license and your intended use is not permitted by statutory regulation or exceeds the permitted use, you will need to obtain permission directly from the copyright holder. To view a copy of this license, visit http://creativecommons.org/licenses/by/4.0/. 

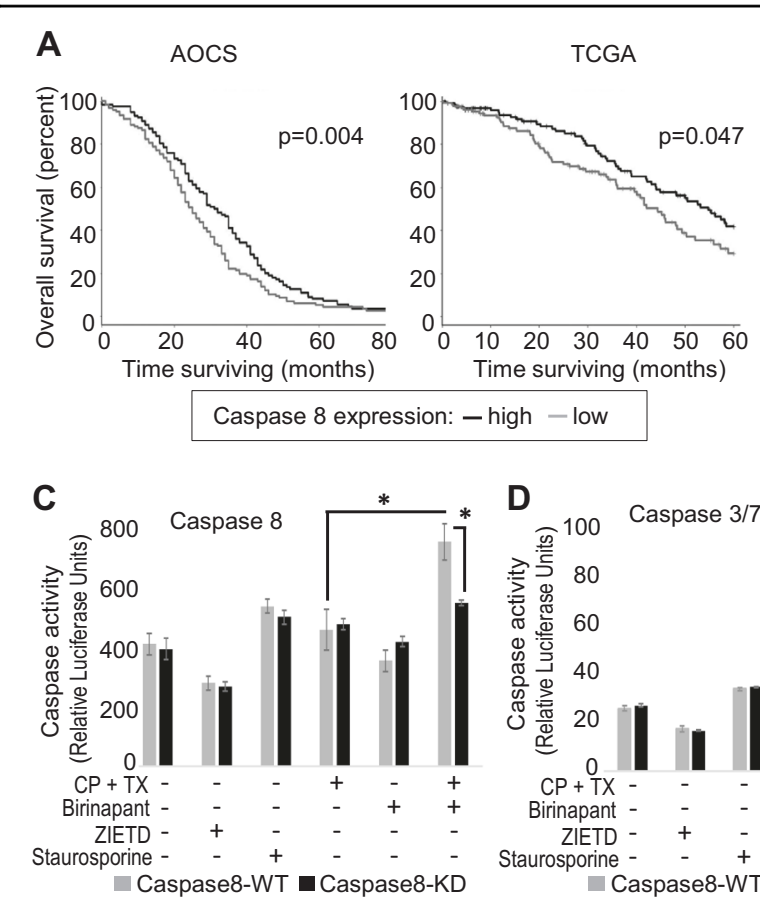

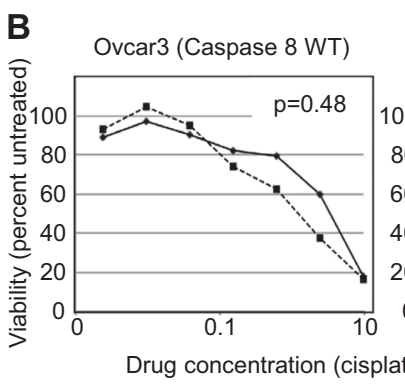

Drug concentration (cisplatin x1 nM, paclitaxel x10 nM)
Ovcar3 (Caspase 8 KD)

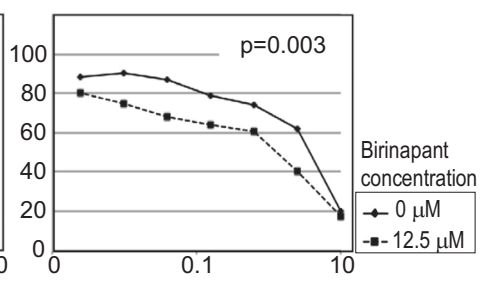

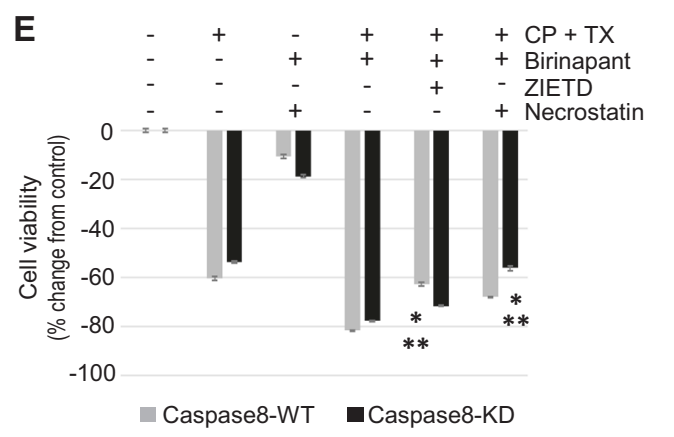

Fig. 1 Low Caspase 8 increases sensitivity of ovarian cancer cell lines to combined chemotherapy treatments. A Overall survival in patients with high (above the median) or low (below the median) Caspase 8 in two datasets shows that low Caspase 8 consistently relates to shorter overall survival. B Ovcar3 cells transduced with either control shRNA (Caspase 8 WT) or Caspase 8 shRNA (Caspase 8 KD) were treated with increasing concentrations of combined cisplatin and paclitaxel in the presence or absence of birinapant (12.5 $\mu \mathrm{M})$ for $72 \mathrm{~h}$. Cell viability was measured by XTT assay. Representative results are expressed as average \pm S.E.M., $n=4$ and shown as percentage of change from vehicle control. $\mathbf{C}$ Ovcar3 cells expressing either control (Caspase $8 \mathrm{WT}$ ) or Caspase 8 shRNA (Caspase $8 \mathrm{KD}$ ) were exposed to combined cisplatin (2.5 $\mu \mathrm{M})$ and paclitaxel (25 nM) and/or Caspase 8 inhibitor ZIETD $(25 \mu \mathrm{M})$, birinapant $(12.5 \mu \mathrm{M})$, and staurosporine $(1 \mu \mathrm{M})$ for $18 \mathrm{~h}$ to induce caspase activity. Results of Caspase 8 cellbased assay are expressed as average luciferase units \pm S.E.M., $n=4$. Single asterisk $\left(^{*}\right)$ represents $p<0.05$ based on $t$ test. D Caspase 3 and 7 activity was measured in the described cell lines and exposed to similar conditions described in $\mathbf{C}$. E Ovcar3 cells expressing either control (caspase 8 WT) or Caspase 8 shRNA (Caspase 8 KD) were exposed for $72 \mathrm{~h}$ to combined cisplatin $(2.5 \mu \mathrm{M})$ and paclitaxel ( $25 \mathrm{nM})$, birinapant, $12.5 \mu \mathrm{M}$, and/or ZIETD $(25 \mu \mathrm{M})$ or NEC1 $(25 \mu \mathrm{M})$ as indicated. Viability was assessed by XTT assay. Results are expressed as average \pm S.E.M., $n=3$. Single asterisk $\left(^{*}\right)$ represents $p<0.01$ based on $t$ test comparing Caspase 8 WT versus KD; Double asterisks $\left(^{* *}\right)$ represent $p<0.01$ based on $t$ test comparing ZIETD to Necrostatin.

apoptotic protein Caspase 8 in ovarian cancer. These studies have shown that patient subgroups expressing high Caspase 8 and high NFKB activity have longer overall survival, but low Caspase 8 and low NFkB activity corresponded with shortest overall survival ${ }^{5}$. We also showed that ovarian cancers depleted of Caspase 8 can be susceptible to cell death by caspase-independent necroptosis 5 . In the current studies, we hypothesized that low Caspase 8 and low $N F \kappa B$ activity can limit apoptosis and decrease the benefit of apoptotic cell death after conventional chemotherapy. We investigated the effect of combined chemotherapy and Smacmimetic treatment as inducers of both apoptotic and necroptotic cell death. In vitro and in vivo, we found ovarian cancer cell death to be similarly pronounced in both Caspase 8 high-expressing and low-expressing ovarian cancers after combined treatment, suggesting that survival benefits may be possible with this therapeutic approach.

\section{Results}

\section{Low Caspase 8 suggests resistance to chemotherapy}

In two large genomic analyses, low Caspase 8 indicated shorter overall survival (Fig. 1A). The Australian Ovarian Cancer Study (AOCS) contains gene expression data from 267 cases, $87 \%$ of which were stage III or IV, and 92\% were serous subtype ${ }^{2}$; The Cancer Genome Atlas (TCGA) profiled 489 cases that were stage II-IV and all were highgrade serous cancers ${ }^{3}$. All patients in both datasets received platinum-based chemotherapy for up-front treatment after tumors were harvested for molecular analyses. In the AOCS and TCGA datasets, expression of Caspase 8 below the median was associated with worse outcome compared to cases with Caspase 8 expression above the median $(p<0.05)$. This suggested poor response to chemotherapy related to lack of Caspase 8 activity. We sought to improve response to chemotherapy in cell lines representative of low Caspase 8 activity. We therefore tested the pair of Ovcar3 cells that we had established, 
with high endogenous Caspase 8 (wild type (WT)) or stable knockdown of Caspase 8 (KD). The matched Ovcar3 cell lines are NFKB-dependent, based on previously published work ${ }^{4}$, and we sought to increase chemotherapy sensitivity by invoking cell death independent of Caspase 8. The SMAC-mimetic birinapant has been shown to induce receptor-interacting protein kinase (RIPK)1-dependent necroptosis ${ }^{6}$. We hypothesized that depletion of cIAP1 with the SMAC-mimetic birinapant would increase the availability of uncleaved RIPK $1^{7}$ and further enhance the effect of Caspase 8 depletion. Interestingly, the addition of birinapant to cisplatin and paclitaxel had little effect on Caspase 8-high cells but increased the sensitivity of Caspase 8-deficient cells especially at low concentrations of chemotherapy (Fig. 1B, $p<0.01$ ). These results suggest a mechanism of either intrinsic apoptosis or necroptosis that bypasses Caspase 8 .

\section{Caspase 8 activity mediates apoptotic cell death of ovarian cancer cells}

We have previously shown that tumor necrosis factoralpha (TNF $\alpha$ ) can promote cell proliferation when cIAP1 is present, as well as induce apoptosis when it is absent $t^{5}$. Caspase 8 is involved in this complex mechanism mediating pro-survival $\mathrm{NF}_{\mathrm{K} B}$ signaling or triggering extrinsic apoptosis downstream of short-term stimulation with TNF $\alpha$ in ovarian cancer cells (Supplementary Fig. 1). We proceeded to investigate whether the apoptotic mechanisms were activated in cells treated with chemotherapy. In this short-term assay, Ovcar3 cells were transduced with Caspase 8 shRNA or control shRNA in order to measure the input of Caspase 8 in chemotherapy-induced cell death signaling. Interestingly, depletion of cIAP1 with the addition of SMAC-mimetic birinapant to the combination chemotherapy did activate Caspase 8 in Ovcar3 cells expressing Caspase 8 , and this rise was attenuated by Caspase 8 depletion (Fig. 1C, " $p<0.05$ ). Assay of Caspase3/7 activity demonstrated rise with cisplatin and paclitaxel, suggesting that these drugs activate intrinsic apoptosis signaling (Fig. 1D). Caspase3/7 activity was augmented with the addition of birinapant $(* p<0.05)$ and was not significantly different in cells with Caspase 8 knocked down $(p=0.16)$. Since Caspase 8-low cells were more susceptible to killing with the combination of chemotherapy and birinapant (Fig. 1B), we asked whether apoptosis or necroptosis contributed to the mechanism of cell death. Cells were treated with the combination of cisplatin, paclitaxel, and birinapant. In Ovcar3 cells with high Caspase 8, inhibition of apoptosis with ZIETD provided relatively more protection than inhibition of necroptosis with necrostatin (Fig. 1E). This effect was reversed in Ovcar3 cells with low Caspase 8, suggesting that cells with low Caspase 8 activity are more likely to die by necroptosis (Fig. 1E, " $p<0.01$ comparing Caspase 8
WT versus KD; $* p<0.01$ comparing ZIETD to Necrostatin). Changes in apoptosis markers cleaved caspase 3 and cleaved poly (ADP-ribose) polymerase (PARP) were assessed by western blot using similar treatment conditions; necroptosis proteins, phosphorylated MLKL, and phosphorylated RIP3 were also measured. Blocking apoptosis with ZIETD again had a more prominent effect in the Caspase 8-WT cells, and inhibition of necroptosis with necrostatin had a greater effect on cell death markers in Caspase 8-KD cells (Supplementary Fig. 2B, C).

\section{Smac-mimetic birinapant combined with carboplatin and paclitaxel increases cell death in NFKB-independent Caspase 8-high and Caspase 8-low cells}

Ovcar3 cells (NFKB dependent) and Ovcar8 cells (NFKB independent) express similar levels of endogenous Caspase $8^{5}$. In order to establish whether the benefit of birinapant addition to chemotherapy was related to NFKB dependence, we investigated whether the patterns of sensitivity to chemotherapy and Caspase 8-independent cell death we observed in Ovcar3 cells would also occur in Ovcar8 cells. We produced a pair of Ovcar8 cells, with high endogenous Caspase 8 or stable knockdown of Caspase 8 (Supplementary Fig. 2). The matched Ovcar8 cell lines showed unique pattern of sensitivity to the combination of carboplatin and paclitaxel; Caspase 8-low Ovcar8 cells were more resistant to carboplatin (higher IC50) than Caspase 8-high cells (Fig. 2A, B). Addition of birinapant to carboplatin significantly increased sensitivity of Caspase 8-high cells over a large range of concentrations (Fig. 2A) but did not similarly affect Caspase 8-low cells (Fig. 2B). Treatment of Caspase 8-high and Caspase 8-low cells with paclitaxel yielded comparable sensitivities, and addition of birinapant increased paclitaxel sensitivity of both cell types at the indicated concentrations (Fig. 2C, D). The results suggest that both

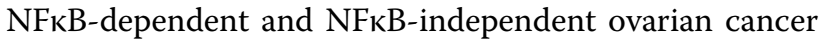
cells can be targeted for additional cell death by chemotherapy and birinapant combined treatment, though the magnitude of effect may differ based on Caspase 8 expression.

\section{Smac-mimetic birinapant synergizes with paclitaxel and antagonizes carboplatin in Caspase 8-depleted cells}

In order to better assess whether a combination treatment of birinapant with carboplatin and paclitaxel together would yield a mathematically synergistic effect, we analyzed the combination indexes (CIs) ${ }^{7}$ of the paired set of Ovcar8 cells treated simultaneously with carboplatin/ birinapant or paclitaxel/birinapant. Caspase 8-high cells showed synergism with a wide dose range of birinapant, combined with each carboplatin and paclitaxel, as indicated by a $\mathrm{CI}<1$. Caspase 8-low cells treated with carboplatin, however, showed moderate antagonism of 


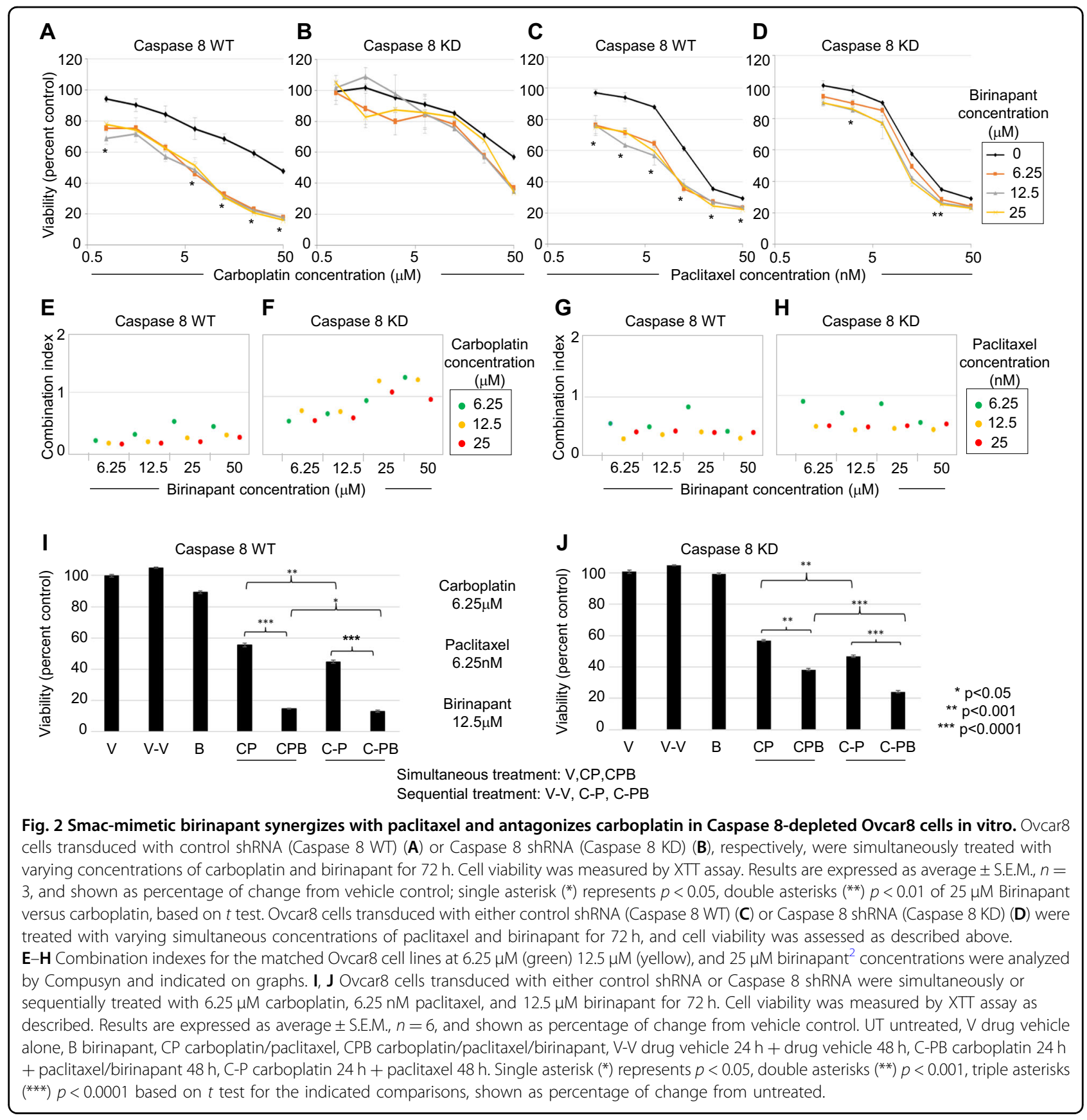

birinapant at high concentrations and mild synergism at lower concentrations. When combined with paclitaxel, birinapant was synergistic over a wide range of concentrations (Fig. 2E-H). These data suggest that in order to achieve the most beneficial synergism between the combination of all three drugs, low concentrations of chemotherapeutic agents as well as birinapant should be used in vitro. Additionally, sequential treatment of paclitaxel-birinapant after carboplatin dosing would avoid any antagonistic effects.
Sequential combination treatment of birinapant and chemotherapy provides maximum cell death benefit to both Caspase-high and Caspase-low cells in vitro

Caspase 8-high and Caspase 8-low cells were exposed to carboplatin, paclitaxel, and low birinapant in synergistic combinations. In order to assure optimal synergistic conditions, simultaneous treatment with the three drugs was compared to sequential treatment, consisting of a 24h treatment with carboplatin, followed by paclitaxel or paclitaxel-birinapant combination, as described. Caspase 
8-high cells significantly benefited from the combined effect of birinapant on chemotherapy, with a 3-4-fold increase in cell death; sequential treatment decreased the level of viability from $15 \%$ following simultaneous treatment to $13 \%$ following sequential treatment (Fig. 2I, ${ }^{*} p<$ 0.05). Caspase 8-low cells also showed a moderate but significant 1.5 -fold enhanced cell death with simultaneous birinapant combined with chemotherapy. Importantly, in these cells with low Caspase 8, sequential treatment significantly enhanced cell death compared to simultaneous exposure to the three drugs, from $38 \%$ viability with simultaneous treatment to $24 \%$ viability with sequential treatment (Fig. 2J, ${ }^{* * *} p<0.001$ ). These results are consistent with the observed antagonism between carboplatin and birinapant and further support the idea that maximum benefit may come from sequential treatment of birinapant and paclitaxel to carboplatin-exposed ovarian cancer cell lines.

\section{Caspase 8-low xenografts grow aggressively, and mice receiving in vivo sequential combination treatment with birinapant and chemotherapy benefit from a delayed rate of tumor growth}

We developed two approaches using our in vitro studies to inform in vivo studies of cooperative effects of birinapant and chemotherapy treatments (Fig. 3A). In a subcutaneous tumor growth model, mice were inoculated with either high Caspase 8 or Caspase 8-depleted cells in order to induce palpable tumors. After 2-3 weeks, mice were randomized and treated with either simultaneous doses of carboplatin, paclitaxel, and birinapant or sequential paclitaxel-birinapant treatments following carboplatin (Fig. 3A). Subcutaneous Caspase 8deficient xenografts were much more aggressive than highCaspase 8 tumors in total growth (Fig. 3B, C), consistent with the patient data showing shortest overall survival in NFkBlow, Caspase 8-low cases ${ }^{5}$. Chemotherapy treatment with or without birinapant decreased tumor growth rate of highCaspase 8 xenografts by $1 / 3$ to $1 / 2$ after 8 weeks (Fig. 3B, " $p$ $<0.05$ compared to vehicle). Chemotherapy-birinapant combinations were most effective in reducing Caspase 8deficient xenografts tumor growth rate by $2 / 3$ after 8 weeks (Fig. 3C, $" p<0.05$ compared to vehicle). Interestingly, simultaneous chemotherapy-birinapant treatment appeared to attenuate tumor reduction, suggesting an in vivo antagonistic effect of birinapant, which was avoided by the sequential dosing schedule.

In separate experiments, we used an orthotopic intraperitoneal inoculation model to mimic advanced-stage ovarian cancer. This model allowed us to investigate whether the above-described effects of treatments would affect overall survival of mice in a model of metastatic, disseminated abdominal tumors. With vehicle treatment, mice bearing Caspase 8-low tumors showed significantly shorter overall survival compared to their Caspase 8-high counterparts, consistent with the increased growth of the Caspase 8-low tumors $(p<0.05)$. High-Caspase 8 xenograft-bearing mice had a 20-30 day improvement on overall survival after treatment with chemotherapy $+1-$ birinapant (Fig. 3D, F). Caspase 8-depleted xenograftbearing mice showed $>30$ days improvement in overall survival when administered chemotherapy $+/-$ birinapant (Fig. 3E, G). Notably, simultaneous treatment of carboplatin and birinapant failed to improve response to chemotherapy alone and significantly reduced the benefit of chemotherapy in Caspase 8-high cells, suggesting an antagonistic effect of birinapant on carboplatin when administered simultaneously in vivo (Fig. 3D, E). In contrast, when carboplatin was administered on day 1 and birinapant was given on days 3 and 5 with paclitaxel treatment, mice with either xenograft experienced significantly longer overall survival than without birinapant (Fig. 3F, G). Again, this highlights consistent synergy of birinapant with paclitaxel but antagonism with carboplatin.

\section{NFKB activity does not follow patterns of birinapant chemo-sensitization in Caspase 8-high or Caspase 8-low cells}

Ovcar3 and Ovcar8 paired cell sets with and without Caspase 8 were studied to assess $\mathrm{NF}_{\mathrm{K}} \mathrm{B}$ activity under synergistic conditions of combined treatments. We investigated the effect of single and combined drug treatments on Caspase 8-high and Caspase 8-low cells transiently expressing an $\mathrm{NFKB}_{\mathrm{K}}$ luciferase reporter construct in the presence or absence of TNF $\alpha$ (Fig. 4). NFkB-dependent Ovcar3 cells with Caspase 8-high and Caspase 8-low expression showed, respectively, 3.5- and 2.5 -fold increased $\mathrm{NF \kappa B}$ activity after birinapant single or combined treatment under unstimulated conditions. Under stimulation with TNF $\alpha$, birinapant did not further increase $\mathrm{NF \kappa B}$ activity in Caspase 8-high cells compared to TNFo stimulation alone, and NFKB activity was 1.5 -fold increased in caspase 8-low cells, likely due to the known role of Caspase 8 in classical NFKB signaling in NFKB-dependent ovarian cells (Fig. 4A). In NFkB-independent Ovcar8 cells with Caspase 8-high and Caspase 8-low, birinapant single and combination treatment resulted in an attenuated but similar 2.3- and 2.4-fold increased activity in steady-state cells, but in a 1.5- and 2.0-fold decreased activities, respectively, in the presence of TNF $\alpha$, compared to TNF $\alpha$ stimulation alone (Fig. 4B). IL-8 secreted level was measured in Ovcar8 cells as a reflection of $\mathrm{NF \kappa B}$ functional activity without TNFa stimulation (Fig. 4C). Unexpectedly, IL-8 levels were higher in Caspase 8-depleted Ovcar8 cells compared to control cells. Birinapant treatment further increased levels of this $\mathrm{NFK}_{\mathrm{B}} \mathrm{B}$ target gene product in Caspase 8-low cells alone and in combination with 


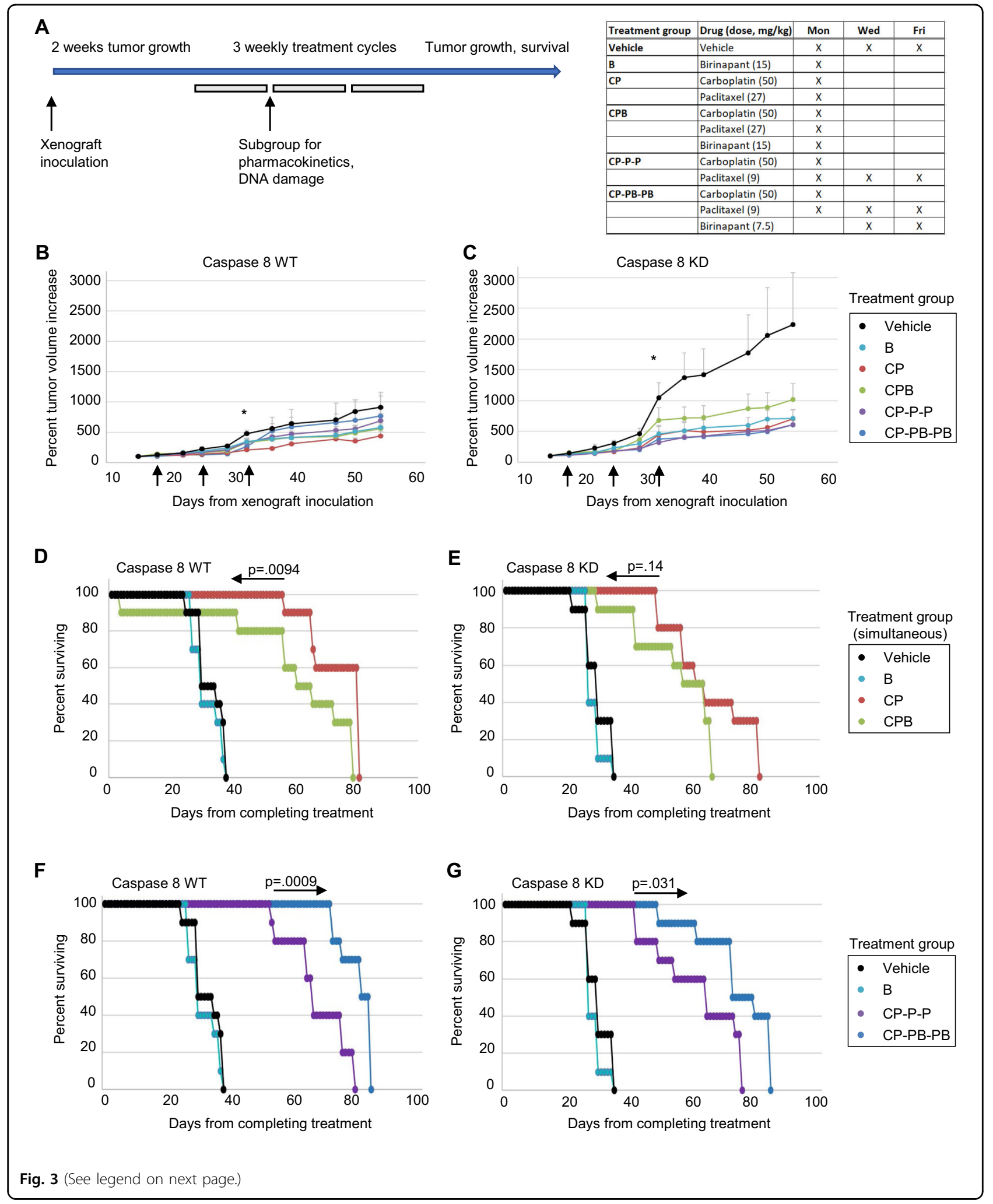


(see figure on previous page)

Fig. 3 In vivo combined chemotherapy-birinapant treatments reduces xenograft growth rate and overcomes carboplatin antagonism.

A Dose and administration schedule for carboplatin, paclitaxel, and birinapant. Mice were inoculated with human ovarian cell lines Ovcar8 with Caspase 8 control (WT) or knockdown (KD). When subcutaneous tumors reached 100-200 $\mathrm{mm}^{3}$ or at 15-20 days for post intraperitoneal inoculations, mice were randomized into groups of 5 . Aqueous solutions of birinapant (Tetralogic, in citrate buffer), carboplatin (Teva), and paclitaxel (Teva) were administered intraperitoneally in $500 \mu \mathrm{l}$ volumes once weekly $\times 3$ weeks or thrice weekly $\times 3$ weeks at the indicated doses. For simultaneous treatments, drug combinations were administered at the same time (CP, CPB), while for sequential treatments, drugs were administered simultaneously on days 1, 3 and 5 (CP-P-P; CP-PB-PB) at doses indicated in the chart. Subcutaneous tumors were measured twice a week as described for 50-60 days or when tumors reached humane endpoints, and IP tumors were assessed after 70-80 days or when health assessments indicated that a humane endpoint had been reached. B Subcutaneous tumor volume increases during and following chemotherapy/birinapant combination treatments of Ovcar8 xenografts expressing wild-type Caspase 8. Points are average of 5 mice; weekly treatments are indicated by black arrows. C Subcutaneous tumor volume increases during and following chemotherapy/birinapant combination treatments of Ovcar 8 xenografts expressing low Caspase 8. Points are average of 5 mice; weekly treatments are indicated by black arrows. Single asterisk (*) represents $p<0.05$, based on $t$ test, and shown as percentage of change from vehicle-treated mice. D-G Overall survival of mice bearing intraperitoneal tumors expressing control (Caspase 8 WT) or low Caspase 8 (Caspase 8 KD), treated with simultaneous $(\mathbf{D}, \mathbf{E})$ or sequential $(\mathbf{F}, \mathbf{G})$ chemotherapy/birinapant administration as described in A. $p$ Values represent two-tail, log-rank comparisons between the indicated survival curves.
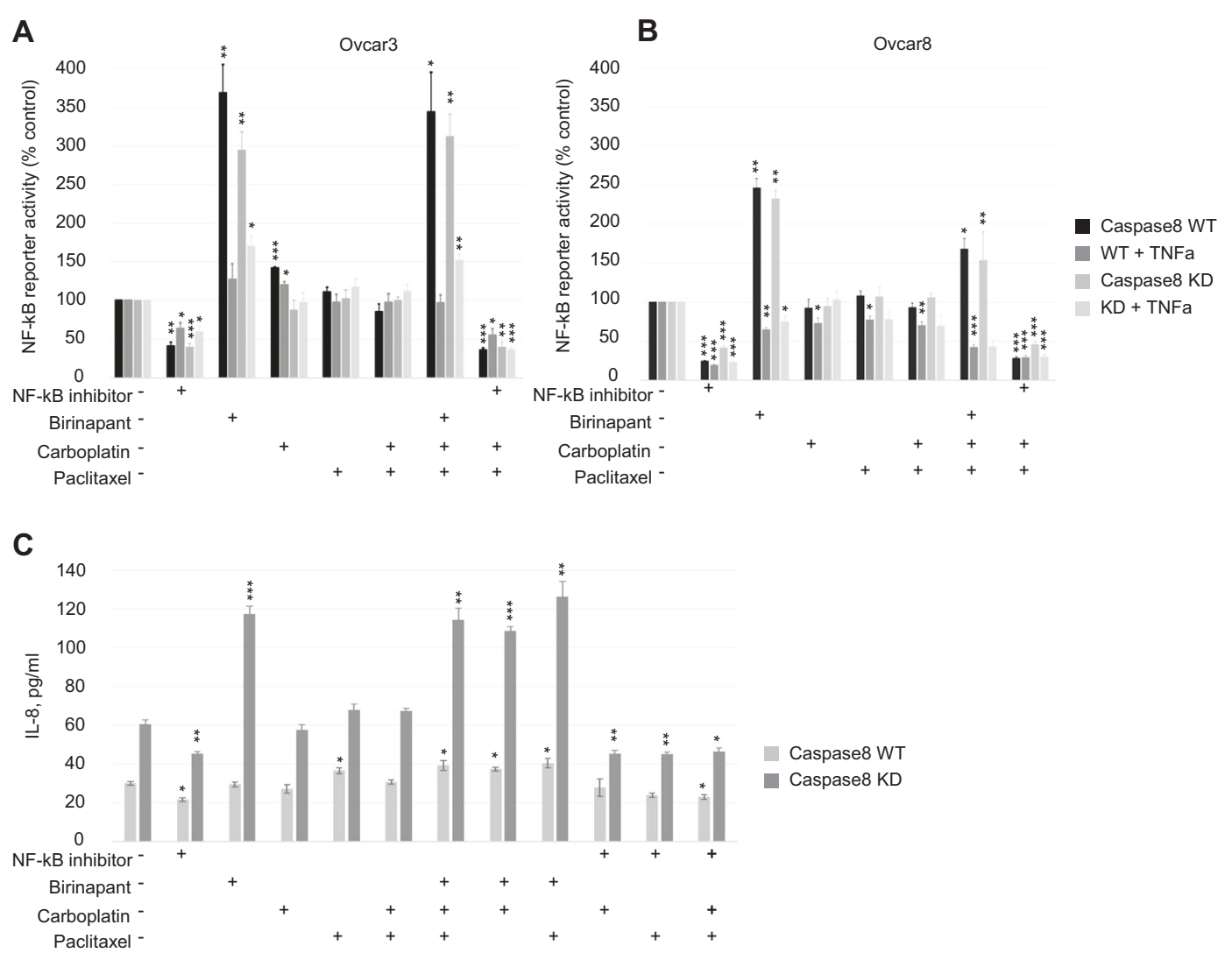

Fig. 4 In vitro birinapant treatment inhibits TNFa-induced NFKB activity in NFKB-independent ovarian cancer cells. A, B NFKB signaling in each Caspase 8 WT and Caspase 8 KD Ovcar3 (A) and Ovcar8 (B) cell lines expressing a reporter was measured with luminescence. Cells were treated with simultaneous combinations of carboplatin, paclitaxel, birinapant, and IKK $\beta$ inhibitor IV for $18 \mathrm{~h}$ as indicated, and TNFa $(10 \mathrm{ng} / \mathrm{ml})$ added for the last hour. Data are arbitrary luciferase units normalized to viable cell number, $n=8$, expressed as percentage of vehicle control. C IL-8 cytokine levels in Ovcar8 Caspase 8 WT and Caspase 8 KD cell lines after treatment with chemotherapy, birinapant, or IKK $\beta$ inhibitor IV for $18 \mathrm{~h}$, as described, $n=8$. Values are $\mathrm{pg} / \mathrm{ml}$.

carboplatin, paclitaxel, or both drugs. Taken together, these experiments suggest that $\mathrm{NF \kappa B}$ activity is unlikely to influence the synergy or antagonism of birinapant with paclitaxel or carboplatin, as no clear distinctions between treatments or cell lines occurred in these assays. 


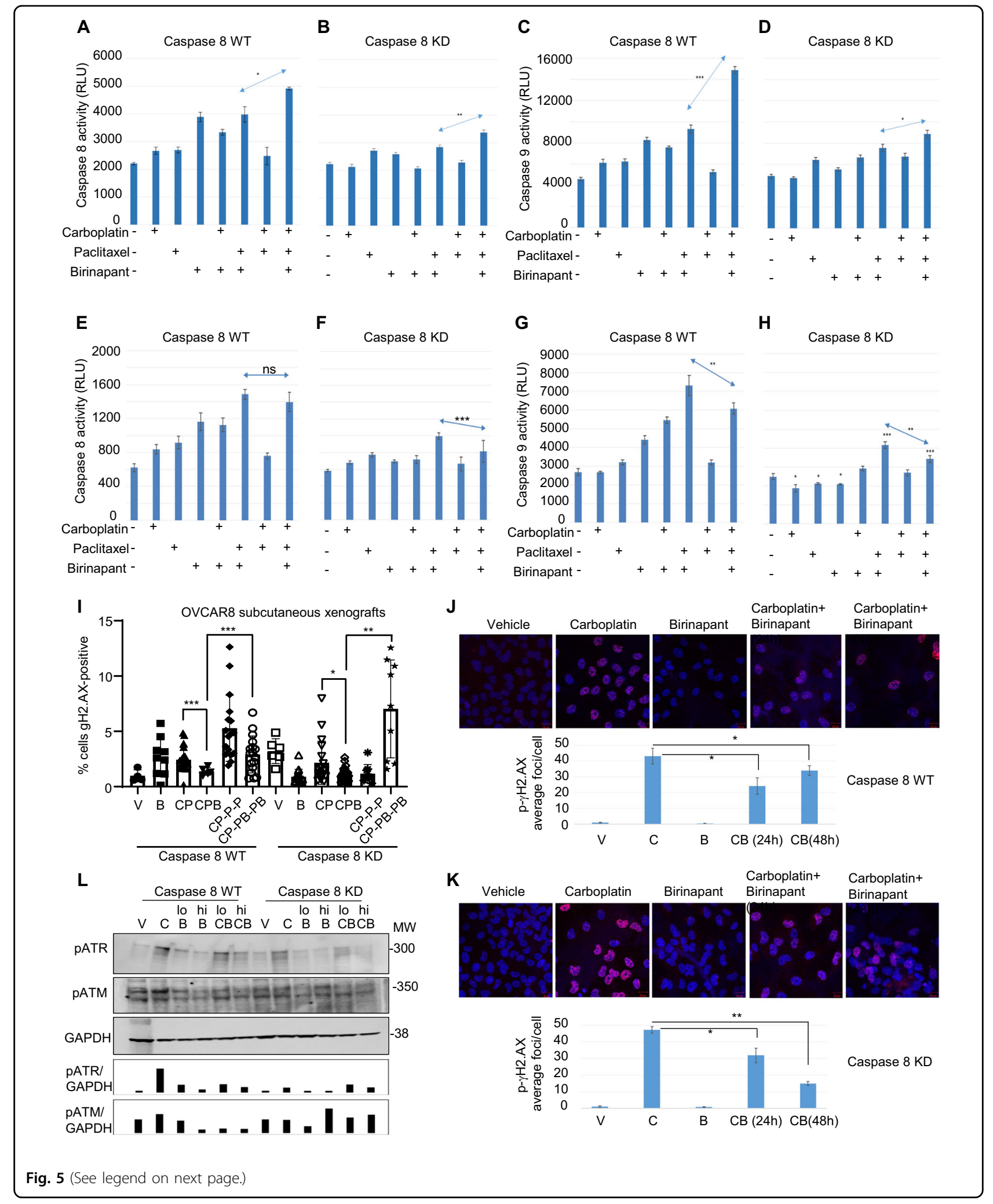


(see figure on previous page)

Fig. 5 Caspase activity response to birinapant/chemotherapy treatment is attenuated by simultaneous administration of carboplatin. Caspase 8 (A, B) and Caspase 9 (C, D) activity was measured after treatment of Caspase 8 WT and Caspase 8 KD cells with carboplatin (6.25 $\mu M)$, paclitaxel $(6.25 \mu \mathrm{M})$, and low Birinapant $(12.5 \mu \mathrm{M})$ for $18 \mathrm{~h}$. Values are arbitrary Caspase units, $n=8$. Single asterisk $\left(^{*}\right)$ represents $p<0.05$, double asterisks $\left.{ }^{* *}\right) p<0.001$, triple asterisks $\left.{ }^{* * *}\right) p<0.0001$ based on $t$ test. E-H Similar experiments were carried out using high Birinapant $(25 \mu \mathrm{M})$. I Percentage of $\mathrm{YH} 2 \mathrm{~A}$.X-positive cells were measured by immunofluorescence in subcutaneous tumors from mice treated as described in Fig. 3. ${ }^{*} p<$ $0.05 ;{ }^{* *} p<0.01 ;{ }^{* * *} p<0.001$. J, K $\gamma H 2 A X$ response was measured in Caspase 8 WT $(\mathbf{J})$ and Caspase 8 KD (K) Ovcar8 cells treated with carboplatin $(6.25 \mu \mathrm{M})$ and/or high birinapant $(25 \mu \mathrm{M})$ simultaneously for $24-48 \mathrm{~h}$ as indicated, using the Zeiss Zen Software from NCl Confocal Core Facility. Data are average $\mathrm{YH}$ 2A.X foci per cell, $n=100$. Single asterisk $\left(^{*}\right)$ represents $p<0.05$, double asterisks $\left(^{* *}\right) p<0.01$, based on $t$ test. $\mathbf{L}$ Western blot analysis of total protein lysates after $18 \mathrm{~h}$ single or simultaneous treatment with carboplatin (C, $6.25 \mu \mathrm{M})$, birinapant (lo B, $12.5 \mu \mathrm{M}$ or hi B, $25 \mu \mathrm{M})$. Loading amounts were assessed by GAPDH staining and ratios of protein to loading control were calculated using ImageJ.

\section{Antagonistic high-dose birinapant combined with} chemotherapy lowers Caspase 9 activity in Ovcar8 cells, suggesting attenuation of intrinsic apoptotic cell death

NFkB-independent Ovcar8 Caspase 8-high and Caspase 8-low cells were treated with simultaneous combinations of carboplatin, paclitaxel, and low (synergistic) or high (antagonistic) birinapant concentrations. Low birinapant concentration increased caspase activity with each single drug as well as combined, in cells expressing high levels of Caspase 8 (Fig. 5A, C); the triple drug combination induced the highest levels of Caspase 8 and Caspase 9 activity, supportive of cell death by extrinsic as well as intrinsic apoptosis. Caspase 8-low cells (Fig. 5B, D) only showed enhancement of caspase activities in paclitaxel single drug or in combination, indicating that triple drug combination was beneficial-albeit to a lesser degree, combining to induce cell death in these chemotherapyresistant cells.

Under high, antagonistic birinapant concentrations, Caspase 8-high cells showed caspase activity induction by single and double combinations, but triple drug combinations did not increase Caspase 8 activity (Fig. 5E) and decreased Caspase 9 activity (Fig. 5G) compared to the combination of birinapant and paclitaxel. Caspase 8-low cells showed minimal significant benefit of double drug combinations and also a decrease in caspase activities under triple combinations (Fig. 5F, H). Taken together, these experiments provide molecular evidence that birinapant synergizes well with paclitaxel under a range of concentrations in cells expressing high and low Caspase 8 levels, while in the presence of carboplatin, only low birinapant concentrations induce increased caspase activities, while high birinapant with carboplatin is detrimental.

\section{Simultaneous birinapant treatment interferes with carboplatin-induced DNA damage}

Carboplatin causes DNA interstrand crosslinks, which lead to single-strand breaks that can be recognized by DNA damage response pathways. Phosphorylated (p)ATR and p-ATM proteins are recruited to strand break sites and phosphorylate $\gamma \mathrm{H} 2 \mathrm{~A} . \mathrm{X}$. We asked whether birinapant interfered with carboplatin DNA damage response. Subcutaneous xenografts were obtained after 1 week of in vivo treatment with simultaneous or sequential doses of birinapant and chemotherapy, as described (schema in Fig. 3A). $\gamma \mathrm{H} 2 \mathrm{~A}$.X staining indicative of DNA damage response was scored. Higher amounts of $\gamma \mathrm{H} 2 \mathrm{~A} . \mathrm{X}$ were present in both Caspase 8-high and Caspase 8-low tumors from mice treated with sequential birinapant-paclitaxel treatment after carboplatin but not simultaneous treatment (Fig. 5I, ${ }^{*} p<0.01$ ). This finding is consistent with our in vivo data showing that the simultaneous presence of birinapant with carboplatin inhibits its antitumor function. These results suggest that birinapant blocks the ability of carboplatin to induce DNA damage, as measured by $\gamma \mathrm{H} 2 \mathrm{~A} . \mathrm{X}$ foci. The presence of birinapant significantly reduced $\gamma \mathrm{H} 2 \mathrm{~A} . \mathrm{X}$ foci in both Caspase 8-high and Caspase 8-low xenografts. The simultaneous administration of birinapant following carboplatin, and with paclitaxel, significantly increased DNA damage compared to the simultaneous administration.

In a separate experiment, Caspase 8-high and Caspase 8-low Ovcar8 cells were treated in vitro with simultaneous combination doses of low and high birinapant and carboplatin for $24 \mathrm{~h}$ and fixed as described. $\mathrm{p}-\gamma \mathrm{H} 2 \mathrm{AX}$ staining (Fig. 5J, K) showed a significant increase in response to single carboplatin treatment and a significant attenuation when administered together with a high dose of birinapant. Based on the kinetics of the measurable changes in a culture of unsynchronized cells, the 24-h timepoint is the most relevant to measure the changes in gamma-H2A.X phosphorylation. The 48-h timepoint was included to show that the effect persists. Levels of pATR and PATM increased in response to single carboplatin treatment of Caspase 8-high and Caspase 8-low Ovcar8 cells, indicating DNA damage response (Fig. 5L). Conversely, these proteins decreased when high-dose birinapant was administered simultaneously with carboplatin. These experiments support the idea that high-dose birinapant administered simultaneously with carboplatin prevents carboplatin-induced DNA damage response as measured by a decrease in phosphorylation and activation of both ATM and ATR proteins and 
decreased $\gamma \mathrm{H} 2 \mathrm{~A}$.X accumulation in DNA. These effects likely attenuate the ability of carboplatin to kill ovarian cancer cells.

\section{Discussion}

Ovarian cancer is recognized to be an extremely heterogeneous disease, presenting unique treatment challenges, and successful therapeutic approaches are increasingly guided by specific genetic and somatic molecular profiles of patient subpopulations in order to maximize clinical benefits. We previously demonstrated that NFKB inhibition can induce cell death by apoptosis in ovarian cancer cells dependent on a TNF $\alpha$-induced pathway for proliferation ${ }^{4}$. More recently, we have shown that Caspase 8 plays a dual role in ovarian cancer, regulating apoptosis and necroptosis downstream of TNF $\alpha 1^{5}$.

In the current study, we show that ovarian cancers expressing low levels of Caspase 8 may be resistant to standard apoptotic-inducing chemotherapy treatments, because they exhibit poorer prognosis than ovarian cancers expressing high Caspase 8 levels. We hypothesized that such patient populations could be targeted by additional SMAC-mimetic treatment in order to deplete cIAP1 and enhance cell death by necroptosis. Using representative ovarian cancer cell lines, we show that the SMAC-mimetic birinapant combined with chemotherapy increased cell death in cells with low Caspase 8, representing the poor prognostic subgroup. Further, in vitro combination treatment with carboplatin, paclitaxel, and low concentration of SMAC-mimetic resulted in synergistic effects that were greater when SMAC-mimetic is administered sequentially, instead of simultaneously, with carboplatin in both high and low Caspase 8 ovarian cancer cells. Using these cell lines to induce xenograft tumors in nude mice, we show that low Caspase 8 cells induced more rapidly growing subcutaneous tumors and that in vivo combination treatments reduced both Caspase 8high and Caspase 8-low tumor growth. The addition of birinapant in the subcutaneous tumor mouse model did not significantly change tumor volume, as all the treatments kept the tumors small in this 8-week timeframe. There are several reasons why the subcutaneous model does not directly mimic the in vitro culture results. Drug delivery to culture is more uniform and likely reaches higher levels when placed directly onto the cells; metabolism of drugs in culture is likely different than metabolism in mouse circulation between peritoneal cavity and subcutaneous capillaries; the mouse subcutaneous tissue microenvironment may also contain other signaling factors that provide chemoresistance, such as cytokines and adhesion molecules.

Long-term survival studies in the orthotopic intraperitoneal mouse model showed significant overall survival benefit with the sequential birinapant treatment in mice bearing either the Caspase 8-high or the Caspase 8-low tumors. Importantly, in this model, the survival of the mice bearing the more aggressive Caspase 8-low ovarian cancer cells reached that of the mice with the Caspase 8high tumors. This suggests that the addition of birinapant to chemotherapy could potentially benefit women with aggressive ovarian cancer if it is added in the correct sequence.

Surprisingly, the presence or absence of NFKB activity did not affect the pattern of cells' response to the combinations of carboplatin, paclitaxel, and birinapant. Birinapant appeared to increase $\mathrm{NF} K \mathrm{~B}$ reporter activity in the absence of TNF $\alpha$, and this was attenuated by the decrease in Caspase 8. Birinapant did not block NFkB signaling in the presence of carboplatin or paclitaxel in either Caspase 8-high or Caspase 8-low cell lines. There was a trend toward decreased NFkB signaling in the NFkB-independent Ovcar8 cell line with the combination of birinapant, carboplatin, paclitaxel, and TNF $\alpha$, perhaps suggesting that the presence of TNF $\alpha$ is a biomarker that should be included in future studies. The NFкB effects, however, were less informative than the apoptosis effects measured in this investigation.

Our studies highlighted that simultaneous administration of standard-of-care carboplatin, paclitaxel, and low concentrations of the SMAC-mimetic birinapant are synergistic with paclitaxel and effective in vitro and in vivo. Higher birinapant concentrations, however, were antagonistic to the carboplatin antitumor effect. The mechanism of SMAC-mimetic interference with carboplatin toxicity after simultaneous administration is not due to lack of Caspase 8 apoptotic activity but likely to an attenuation of carboplatin-induced DNA damage and the DNA repair response. Sequential administration of birinapant avoided the antagonistic effect. Taken together, carboplatin, paclitaxel, and birinapant combination treatments are synergistic and result in additional Caspase 8 -independent tumor cell death and could benefit patient subpopulations who are resistant to apoptotic treatments. This work highlights the importance of understanding mechanisms of drug action in order to design optimal treatment schedules.

\section{Conclusions}

Improved pathway-directed therapeutic advances are needed in order to improve ovarian cancer clinical outcome. Ovarian cancer populations exhibiting chemoresistance likely have defects in apoptotic signaling and circumvent treatments designed to induce tumor cell death by this mechanism. To enhance standard-ofcare chemotherapy treatment of ovarian cancer, a tailored combination treatment of carboplatin, paclitaxel, and the SMAC-mimetic birinapant can enhance tumor 
cell death in vitro and in vivo, suggesting a useful, selective therapeutic benefit to specific patient subpopulations.

\section{Materials and methods Cell viability assays}

Ovcar3 and Ovcar8 cells were previously described ${ }^{4}$ and represent PARP inhibitor-resistant ovarian cancer. All ovarian cell lines were authenticated by Short Tandem Repeat Analysis performed by the Molecular Detection Group, SAIC, Frederick National Laboratory, in reference to the ATCC profile for the same cell line (www.atcc.org). Adherent ovarian cancer cell viability was assessed using XTT (Sigma-Aldrich) as described ${ }^{8}$. Briefly, cells were seeded in 96-well plates at a density of $1-2000$ cells $/ 50 \mu \mathrm{l} /$ well and incubated for $24 \mathrm{~h}$. To analyze the lethal effects of cisplatin, carboplatin, paclitaxel, or birinapant on ovarian cancer cells, cells were Caspase 8 depleted using a previously validated short hairpin RNA (shRNA) clone ${ }^{5}$ or control scrambled shRNA. Cisplatin, carboplatin, and paclitaxel were from Tocris; birinapant was from Tetralogic, through a Materials Transfer Agreement ${ }^{5}$. All drugs were prepared as dimethyl sulfoxide (DMSO) stocks and serially diluted in RPMI medium to $2 \times$ concentrations, then added in $50 \mu \mathrm{l}$ aliquots to each cell well. Final DMSO concentrations did not exceed $0.5 \%$, previously established to be non-toxic to the cells. Plates were incubated for up to 10 days, and inhibitors replenished every 3-4 days. Cell viability was assessed by incubating cultures with $\mathrm{XTT}^{8}$ and absorbances read in a Spectramax M5 plate reader (Molecular Devices). Cell density in treated wells was expressed as a percentage of vehicle-treated control wells. Experiments included 3-6 replicate samples per point and were repeated at least three times. All viability studies were performed according to the above format, using $25 \mu \mathrm{M}$ Caspase 8 inhibitor ZIETD (Z-IETD-FMK, FMK-007, R\&D Systems), $20 \mu \mathrm{M}$ NEC1 (Necrostatin-1, Tocris), or birinapant added to cells either alone or in combination, as described.

\section{Caspase activity assays}

Caspase 8 and Caspase 3/7 activities were measured using cell cultures treated with chemotherapy, birinapant, or inhibitors for $18 \mathrm{~h}$ as described ${ }^{5}$. Activity data were normalized to viable cell number obtained by XTT assay, on duplicate assay plates. As in viability assays, experiments included 3-6 replicate samples per point and were repeated at least three times. Caspase 8 high (wild-type, WT) and Caspase 8 low (knockdown, KD) ovarian cancer cells were analyzed by cell-based Caspase 8 and Caspase 9 (G8200, G8090, G8210) luminescence assays (Promega), according to the manufacturer's specifications.

\section{Patient datasets}

Geo Dataset 9899 contains the gene expression for the AOCS samples ${ }^{2}$. Data were $\log 2$ transformed prior to calculation. The TCGA data portal is found at https:// tcga-data.nci.nih.gov/docs/publications/ov_2011/ ${ }^{3}$.

Expression data in the portal have been normalized to non-cancer controls. Gene expression data were grouped into designated subtypes, as per the respective publications, and the average expression of Caspase 8 and $\mathrm{NFKB}$ gene signature was calculated. The NFkB gene signature is previously published ${ }^{4}$. Kaplan-Meier survival plots were generated using online calculator at https://statcom.dk/KM_plot.

\section{Xenograft experiments}

Ovcar8 and Ovcar3 cell lines were obtained and maintained as described before ${ }^{9}$. Cells were authenticated via Short Tandem Repeat analysis by the Molecular Detection Group, Leidos, Frederick National Laboratory, in reference to the ATCC (www. atcc.org) and Cell Miner (http://discover.nci.nih.gov/ cellminer) profile for the same cell line. Cells used for tumor induction were tested by MTBM as required by the NCI ACUC Committee and confirmed to contain no mouse viruses. Cells $\left(1-2 \times 10^{6}\right)$ were counted and freshly prepared as suspensions in $0.5 \mathrm{ml}$ phosphatebuffered saline for subcutaneous (flank) or intraperitoneal injections into 6-8-week-old athymic nu/nu female mice in randomly assigned groups of 10 mice. Drug treatments were administered intraperitoneally as described, freshly prepared in aqueous $0.5 \mathrm{M}$ sodium citrate buffer $\mathrm{pH}$ 5.0. Mice were housed and observed according to approved NCI-ACUC guidelines. Total body weights and subcutaneous tumor caliper measurements were taken twice weekly in mice exhibiting palpable subcutaneous tumors until 5-6 weeks or humane endpoints. Subcutaneous tumor volumes were calculated according to the formula $V=1 / 2$ (length $\times$ width $\left.^{2}\right)$; tumor burden for mice injected intraperitoneally was assessed by monitoring body weight and histopathology analysis after 6-10 weeks or humane endpoints. Survival studies assessed mouse survival at experimental humane endpoints. Veterinary staff were blinded to treatment group. Statistical analysis of survival studies was done by log-rank test.

\section{Compusyn analysis}

Analysis of dose effects after combination treatments of cells with carboplatin or paclitaxel with birinapant was performed using the CompuSyn for Drug Combinations and for General Dose-Effect Analysis software, as described $^{10}$. 


\section{NFkB activity reporter assays}

Ovcar3 and Ovcar8 cells subjected to scrambled or Caspase 8 shRNA knockdown were first selected with puromycin as described before ${ }^{4}$. After selection, cells were transduced with a lentiviral vector containing an $\mathrm{NF \kappa B}$ transcriptional regulatory element, using the Cignal Lenti Reporter System (CLS-013L), according to the manufacturer's specifications and allowed $72 \mathrm{~h}$ for maximum vector expression. Transient reporter assays were subsequently performed for $18 \mathrm{~h}$. Briefly, cells were plated in 96-well plates at a density of 10,000 cells/ well. After overnight attachment, cells were exposed to serum starvation medium containing $0.5 \%$ fetal bovine serum for $24 \mathrm{~h}$. Drugs or IKK $\beta$ inhibitor IV (EMD Biosciences) were added for $1 \mathrm{~h}$ after which TNF $\alpha$ (30001A, PeproTech) was added to stimulate NFkB activity for $18 \mathrm{~h}$. Control wells received vehicle alone. Luciferase activity was measured using the Luciferase Assay System (E4030, Promega) according to the manufacturer's instructions and a Spectramax M5 plate reader (Molecular Devices). Luciferase units were normalized to viable cell number, obtained by XTT assay, on duplicate assay plates. Experiments included 3-6 replicate samples per point and were repeated at least three times.

\section{IL-8 mesoscale cytokine analysis}

Ovcar8 cells expressing WT or low Caspase 8 levels were subjected to treatment with carboplatin, paclitaxel, birinapant, and NFKB inhibitor single or combined simultaneous treatments, for $18 \mathrm{~h}$ as described. Culture supernatants $(100 \mu \mathrm{l})$ were collected after 18 -h drug treatment and assessed for the levels of IL-8 using Mesoscale Kit (MSD K15025B) according to the manufacturer's specifications. Experiments included eight replicate samples per cytokine and were repeated twice.

\section{$\mathrm{p}-\mathrm{\gamma H} 2 \mathrm{AX}$ immunohistochemistry assay}

Ovcar8 cells expressing WT or low Caspase 8 levels were subjected to treatment with carboplatin, birinapant, or combined simultaneous treatment with carboplatin and antagonistic dose of birinapant for $18 \mathrm{~h}$. Cells were fixed with $4 \%$ paraformaldehyde and standard immunohistochemistry was performed to assess the levels of phosphorylated $\gamma \mathrm{H} 2 \mathrm{AX}$ as a measure of carboplatin-induced DNA damage. Subcutaneous xenograft tumors from mice treated with single, simultaneous, and sequential drug combinations were freshly fixed after a week of treatment and subjected to phosphorylated $\gamma \mathrm{H} 2 \mathrm{AX}$ level assessment using the same antibody ( $\mathrm{p}-\gamma \mathrm{H} 2 \mathrm{AX}$ antibody Millipore \# 05-636-AF488). Experiment included ten mice per group.

\section{BOND-max Autostainer Staining/image capture and quantitation}

The method used for staining formalin-fixed paraffinembedded tissue sections was modified from our previously described Bond-max ${ }^{\mathrm{TM}}$ Autostainer Staining protocol ${ }^{11}$. The following antibody working solutions were prepared and loaded into the BOND-MAX Processing Module: $10 \mu \mathrm{g} / \mathrm{ml}$ monoclonal cleaved caspase-3 (R\&D systems, MAB835, clone 269518, Milan, Italy) antibody prepared in Bond Primary Antibody Diluent (Leica Biosystems, Buffalo Grove, IL) and goat-anti-rabbit Alexa Fluor 546 (Invitrogen, Life Technologies, Grand Island, $\mathrm{NY}$ ) antibody prepared 1:100 in $1 \times$ Bond Wash Solution; $5 \mu \mathrm{g} / \mathrm{ml}$ monoclonal anti-phospho (Ser 139) $\gamma \mathrm{H} 2 \mathrm{AX}-$ FITC conjugate (Millipore, 16-202a, clone JBW301, Billerica, MA) antibody was prepared in Bond Primary Antibody Diluent (Leica Biosystems, Buffalo Grove, IL); and DAPI dihydrochloride, FluoroPure ${ }^{\mathrm{TM}}$ grade (Invitrogen, Life Technologies, Grand Island, NY) prepared at $0.25 \mu \mathrm{g} / \mathrm{ml}$ in Bond Primary Antibody Diluent. Slides were cured overnight with Prolong Gold Antifade Reagent (Invitrogen) in the dark and imaged the following day. For long-term storage, slides were stored in the dark at $-20^{\circ} \mathrm{C}$.

Slides stained with the cleaved caspase-3/ $\gamma \mathrm{H} 2 \mathrm{AX} / \mathrm{DAPI}$ multiplex and were scanned using the Aperio ScanScope FL image capture system (Leica Biosystems) for each xenograft or biopsy with at least six fields from each slide analyzed. Images were acquired using $\times 20$ magnification with a Leica Plan Apo 20X/0.7NA objective, $0.4622 \mu \mathrm{m} /$ pixel resolution, and images had a 16-bit depth. The cleaved caspase- 3 exposure was $1.6 \mathrm{~s}, \gamma \mathrm{H} 2 \mathrm{AX}$ exposure of $500 \mathrm{~ms}$, and DAPI exposure $80 \mathrm{~ms}$. Images were extracted with a fixed size $(1000 \times 1000$ pixel $)$ from full tissue scans using the Aperio ImageScope software. Images that were selected for extraction were derived from tumor tissue determined to be of sufficient quality as assessed during the evaluation of an adjacent hematoxylin and eosin-stained section of each specimen and necrotic regions of the tissue were avoided. Definiens Architect Tissue Studio IF software (Definiens AG, Munich, Germany) was used for quantitative analysis for $\gamma \mathrm{H} 2 \mathrm{AX}$ biomarker expression by percentage of $\gamma \mathrm{H} 2 \mathrm{AX}$-positive nuclei analysis and nuclear segmentation and enumeration of all xenograft material. Additional analysis methods are described previously ${ }^{11}$.

\section{Western analysis}

Total protein was extracted from described ovarian cell cultures expressing Caspase 8 or scrambled shRNA untreated, treated with chemotherapy, and/or birinapant using RIPA buffer (sc-24948) according to the manufacturer's protocol (Santa Cruz Biotechnology, Santa Cruz, CA) and concentrations were estimated with the 
BCA Protein Assay Kit (Thermo Scientific, Rockford, IL). Sodium dodecyl sulfate-polyacrylamide gel electrophoresis and western blot analysis were performed using, respectively, the NuPage system (Invitrogen, Carlsbad, CA) and the Supersignal Chemiluminescent Substrate system (Thermo Scientific, Rockford, IL) using PhosphoATM (Cell Signaling \#4526), Phospho ATR (Abcam \#ab227851), and GAPDH (Abcam \#ab9485) antibodies.

\section{Statistical analysis}

All in vitro experiments were performed a minimum of three independent experiments, with 3-8 technical replicates per experiment. Replicate numbers were maximized to ensure adequate power for assessing differences between groups. Statistical comparisons of significance were done by one-way analysis of variance with Tukey post hoc adjustment for multiple comparisons for viability, Caspase activity, and NFkB activity assays, as well as p-gH2.AX in vitro counts. Normal distribution and equal variance were assumed in the computations. Mouse survival curves were compared using Kaplan-Meier log-rank tests. With 10 mice per group, we sought at least $90 \%$ power to detect an effect size of 1.0 with alpha 0.1. Significance and $p$ values comparisons are described in the figure legends.

\section{Acknowledgements}

We thank Elena Kuznetsova and Geneti Gaga for expert mouse xenograft experiment assistance and Diana Haines (Leidos Biomedical Research, Inc. Frederick National Laboratory for Cancer Research) for expert pathology assessment of abdominal xenograft occurrence and severity in mouse studies. The authors would like to acknowledge Brad Gouker and Donna Butcher Leidos Biomedical Research, Inc. for their technical contribution to histology efforts.

\section{Author contributions}

Conception and design: C.M.A. Development of methodology: L.F.H. Acquisition of data: L.F.H. (in vitro assays, cell culture, mouse models, data collection and analysis), A.B.D. (immunofluorescence data collection and analysis), S.K. (in vitro assays), C.M.A. (human data set analysis). Analysis and interpretation of data: (statistical analysis, biostatistics), C.M.A. Writing, review, and/or revision of the manuscript: L.F.H., S.K., C.M.A. Administrative, technical, or material support: C.M.A. Study supervision: C.M.A.

\section{Funding}

This project was funded by the National Cancer Institute Intramural Program (CMA), with federal funds from the National Cancer Institute, National Institutes of Health, under Contract No. HHSN261200800001E (MA). The content of this publication does not necessarily reflect the views of policies of the Department of Health and Human Services, nor does mention of trade names, commercial products, or organizations imply endorsement by the U.S. Government. Open Access funding provided by the National Institutes of Health $(\mathrm{NIH})$.

\section{Conflict of interest}

The authors declare no competing interests. Birinapant was obtained under a Materials Transfer Agreement with the National Cancer Institute and Tetralogic Pharmaceuticals.

\section{Publisher's note}

Springer Nature remains neutral with regard to jurisdictional claims in published maps and institutional affiliations.

Supplementary information The online version contains supplementary material available at https://doi.org/10.1038/s41420-021-00511-2.

Received: 8 January 2021 Accepted: 5 May 2021

Published online: 04 June 2021

\section{References}

1. Siegel, R. L., Miller, K. D. \& Jemal, A. Cancer statistics, 2020. CA Cancer J. Clin. 70, 7-30 (2020).

2. Tothill, R. W. et al. Novel molecular subtypes of serous and endometrioid ovarian cancer linked to clinical outcome. Clin. Cancer Res. 14, 5198-5208 (2008).

3. Cancer Genome Atlas Research Network. Integrated genomic analyses of ovarian carcinoma. Nature 474, 609-615 (2011).

4. Hernandez, L. et al. Activation of NF-kappaB signaling by inhibitor of NFkappaB kinase beta increases aggressiveness of ovarian cancer. Cancer Res. 70, 4005-4014 (2010).

5. Hernandez, L. et al. A dual role for Caspase8 and NF-kappaB interactions in regulating apoptosis and necroptosis of ovarian cancer, with correlation to patient survival. Cell Death Discov. 1, 15053 (2015).

6. Benetatos, C. A. et al. Birinapant (TL32711), a bivalent SMAC mimetic, targets TRAF2-associated CIAPs, abrogates TNF-induced NF-kappaB activation, and is active in patient-derived xenograft models. Mol. Cancer Ther. 13, 867-879 (2014).

7. Laukens, B. et al. Smac mimetic bypasses apoptosis resistance in FADD- or caspase-8-deficient cells by priming for tumor necrosis factor alpha-induced necroptosis. Neoplasia 13, 971-979 (2011).

8. Scudiero, D. A. et al. Evaluation of a soluble tetrazolium/formazan assay for cell growth and drug sensitivity in culture using human and other tumor cell lines. Cancer Res. 48, 4827-4833 (1988).

9. Hernandez, L. et al. Characterization of ovarian cancer cell lines as in vivo models for preclinical studies. Gynecol. Oncol. 142, 332-340 (2016).

10. Chou, T. C. \& Talalay, P. Quantitative analysis of dose-effect relationships: the combined effects of multiple drugs or enzyme inhibitors. Adv. Enzyme Regul. 22, 27-55 (1984).

11. Dull, A. B. et al. Development of a quantitative pharmacodynamic assay for apoptosis in fixed tumor tissue and its application in distinguishing cytotoxic drug-induced DNA double strand breaks from DNA double strand breaks associated with apoptosis. Oncotarget 9, 17104-16. (2018). 